\title{
Embedded Signal Approach to Image Texture Reproduction Analysis
}

\author{
Peter D. Burns a and Donald Baxter ${ }^{\mathrm{b}}$ \\ ${ }^{a}$ Burns Digital Imaging, Fairport, NY USA \\ ${ }^{\mathrm{b}} \mathrm{STMicroelectronics,} \mathrm{Edinburgh,} \mathrm{UK}$
}

\begin{abstract}
Since image processing aimed at reducing image noise can also remove important texture, standard methods for evaluating the capture and retention of image texture are currently being developed. Concurrently, the evolution of the intelligence and performance of camera noise-reduction (NR) algorithms poses a challenge for these protocols. Many NR algorithms are 'content-aware', which can lead to different levels of NR being applied to various regions within the same digital image. We review the requirements for improved texture measurement. The challenge is to evaluate image signal (texture) content without having a test signal interfere with the processing of the natural scene. We describe an approach to texture reproduction analysis that uses embedded periodic test signals within image texture regions. We describe a target that uses natural image texture combined with a multi-frequency periodic signal. This low-amplitude signal region is embedded in the texture image. Two approaches for embedding periodic test signals in image texture are described. The stacked sine-wave method uses a single combined, or stacked, region with several frequencycomponents. The second method uses a low-amplitude version of the IEC-61146-1 sine-wave multi-burst chart, combined with image texture. A $3 \times 3$ grid of smaller regions, each with a single frequency, constitutes the test target. Both methods were evaluated using a simulated digital camera capture-path that included detector noise and optical MTF, for a range of camera exposure/ISO settings. Two types of image texture were used with the method, natural grass and a computed 'dead-leaves' region composed of random circles. The embedded-signal methods tested for accuracy with respect to image noise over a wide range of levels, and then further in an evaluation of an adaptive noise-reduction image processing.
\end{abstract}

Keywords: image quality, texture MTF, camera performance, stacked sine-wave, grid multi-burst, Siemens star

\section{INTRODUCTION}

Image texture is the term given to the signal (information-bearing) fluctuations such as those for skin, grass and fabrics. Since image processing aimed at reducing image noise can also remove important texture, standard methods for evaluating the capture and retention of image texture are currently being developed. Concurrently, the evolution of the intelligence and performance of camera noise-reduction (NR) algorithms poses a significant challenge for these protocols. Algorithms already exist for which the Dead Leaves [1] and low contrast radial sine-wave texture reproduction protocols [3] ratings are inconsistent with subjective texture quality. This paper reviews the requirements for an improved texture measurement protocol, and describes an alternative approach to texture reproduction analysis. This method is based on embedding periodic test signals within texture regions.

Many of the latest NR algorithms are 'content-aware', which can lead to different levels of NR being applied to various regions within the same digital image. The non-local (NL) class of NR algorithms [4] look for pixels with a similar local neighborhood within a larger search window. This approach dynamically finds the best region, line or curve over which to filter image pixel variations. This form of local decision-making reduces the effectiveness of the Dead Leaves texture evaluation method, in particular the separation of image (signal) texture fluctuations from noise, estimated from uniform areas. [5] Other methods for texture capture evaluation are also influenced by these noise-reduction methods. A radial sine-wave target, also called a Siemens Star, is used to evaluate an effective signal MTF. However, some noise-reduction algorithms filter noise along the radial fingers of the target, which can lead to misleading results (Figure 1).

Proc. SPIE Vol. 9016, Image Quality and System Performance XI (2014) 

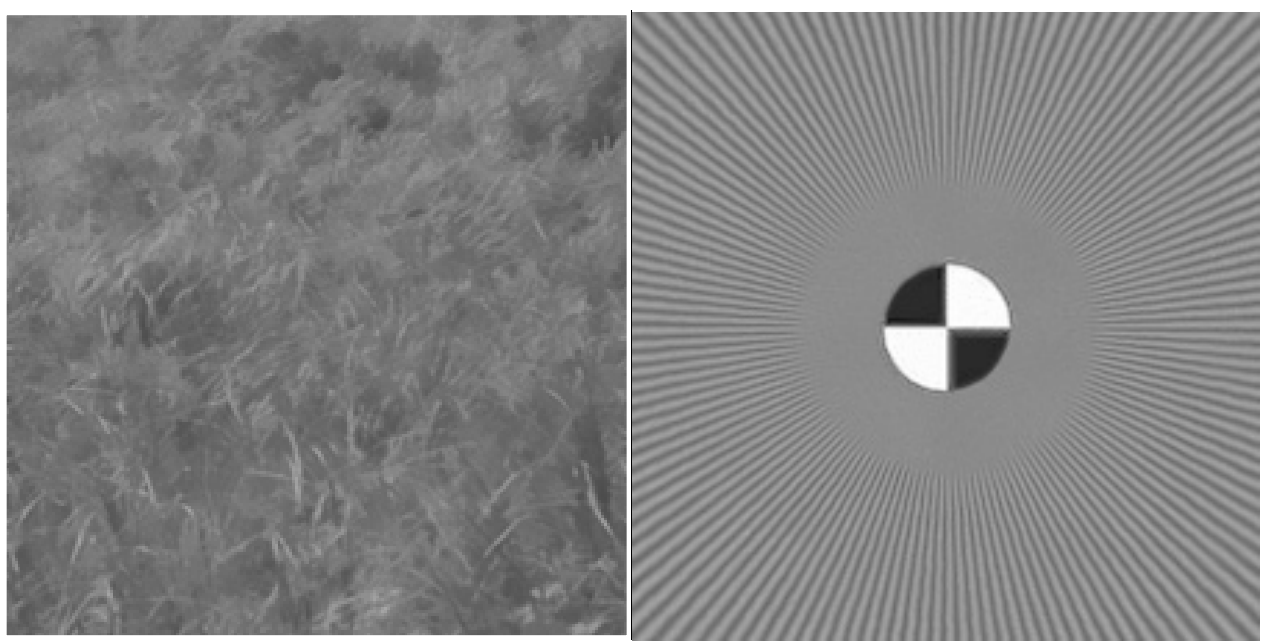

Figure 1 : ISO 25,600 grass (left) and Siemens Star (right) images, processed with the same non-local means filter strength (665.6). Note that the grass is blurred but the low contrast Siemens Star frequencies $<0.4$ cycles/pixel are not. The Siemens Star input image for the non-local means filter had a constant contrast ( $9 \%$ amplitude) up to 0.4 cycles/pixel.

In developing an improved method for measuring the capture of image texture the following requirements were considered;

- The test target should have texture fluctuations similar to natural materials such as grass, to ensure realistic NR algorithm behavior.

- The analysis should result in a reliable texture MTF in the presence of image noise such as that observed with current equipment and high ISO (low-light) settings.

- The influence of non-linear signal mapping should be minimal.

Figure 2 summarizes the various steps followed in this paper. We describe two approaches to embedding periodic signals in image texture. In each case we consider the test target and subsequent analysis that is required for the spatialfrequency based resolution measure, similar to the modulation transfer function (MTF). Both methods are then applied to test images generated via a camera simulation for a range of exposure and ISO camera settings. This is done in order to evaluate the stability of the measures in the presence of image noise. Following this verification, we apply the embedded-signal analyses to the case of non-local noise suppression image processing.

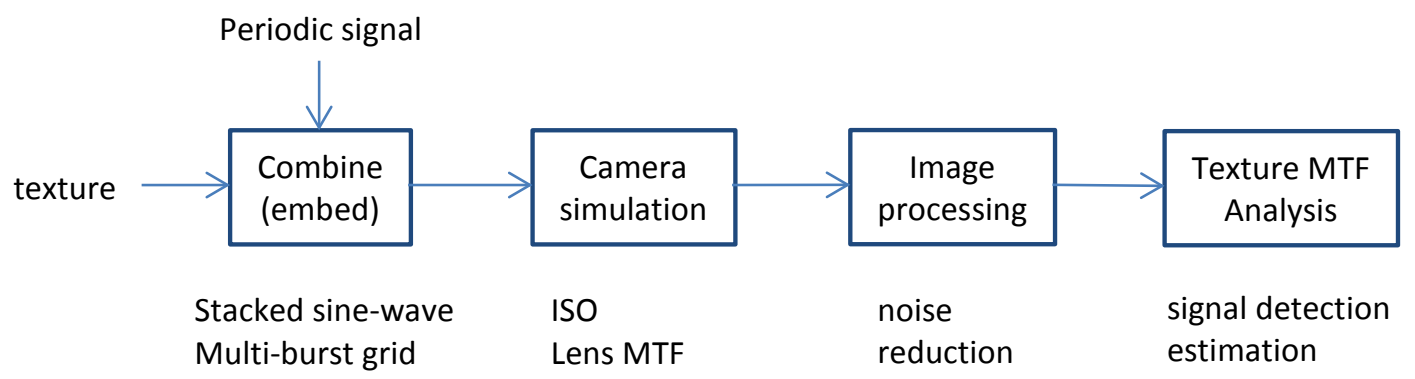

Figure 2 : Outline of the embedded-signal method and testing that we will describe

We have simplified our analysis and presentation of results by focusing on monochrome, grayscale, images. In addition, we include comparisons with an established sine-wave method for imaging system evaluation, based on a polar sinewave pattern, the continuous-tone Siemen's Star. 


\section{STACKED SINE-WAVE METHOD}

\subsection{Test Chart}

This method relies on the combination of several one-dimensional periodic signals, each with a different spatial frequency. By choosing a single region for all components, we increase the chance of detection of the periodic components when they are presented with the 'background' image texture. Periodic signals have discrete Fourier components, rather than the continuous spectra of stochastic processes. If we maximize the data size, or record area/length, we increase the chance that the discrete components can be isolated using a frequency-based detection and analysis method. Figure 3 shows an example of the summation of six components, each computed with a random phase.
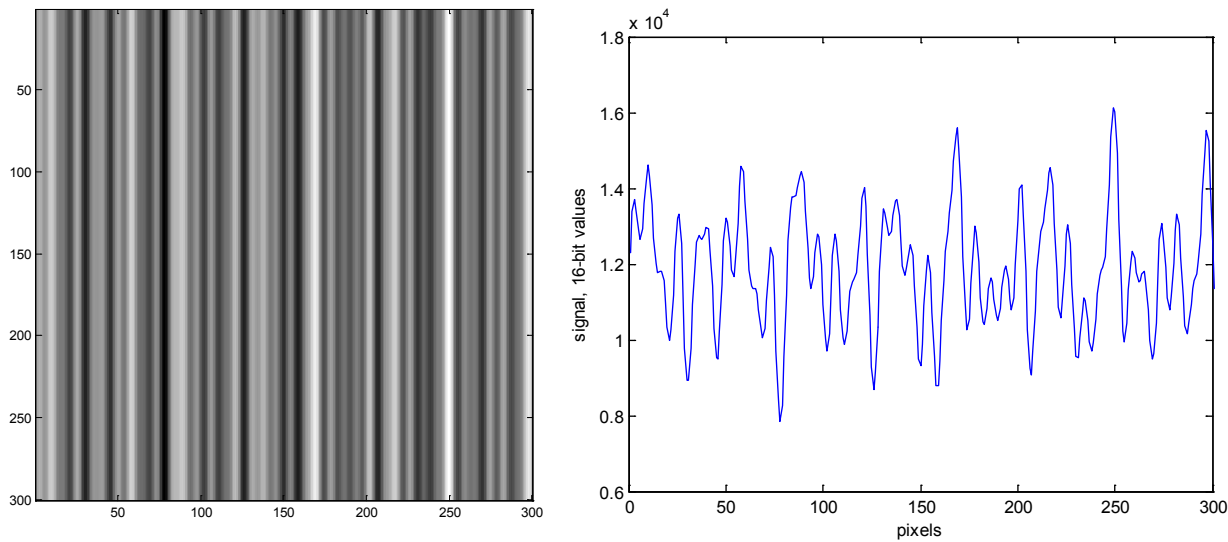

Figure 3: Six-frequency stacked sine-wave region and a cross-section, computed for a 16-bit encode test image. The image in the left is displayed with a higher contrast than used in practice.

For an image array of width $M$ pixels and set of $N$ frequencies $\left\{f_{1}, \ldots, f_{N}\right\}$, the one-dimensional signal is computed as,

$$
s_{j}=a \sum_{n=1}^{N} \sin \left(2 \pi f_{n} j+\phi_{n}\right), j=1, \ldots, M
$$

where $\left\{\phi_{1}, \ldots, \phi_{N}\right\}$ are the set of random phase values. This vector is then replicated to for a digital image region, as show on the left of Figure 3.

\subsection{Analysis Method}

Various methods are available to us for the detection and estimation of the amplitude of the embedded periodic signal components in our test image region. Several, for example the MUSIC (Multiple Signal Classification) algorithm, [6] take advantage of a priori knowledge of the number of sine-wave components. For our detection and analysis of the stacked sine-waves, however, we used the computationally simpler Discrete Fourier Transform followed by peakdetection applied to the modulus vector. The for an ( $\mathrm{M} \mathrm{x} \mathrm{M}$ ) array, $s$, modulus is given by,

$$
U(p, q)=\left|\sum_{x=M / 2+1}^{M / 2} \sum_{y=M / 2+1}^{M / 2} s(x, y) e^{-2 i \pi(p x+q y)}\right| .
$$

When the one-dimensional components are sinusoidal oriented along the $\mathrm{x}$ - or $\mathrm{y}$-axis, as they were for our study, the two-dimensional DFT is first computed. The zero-frequency cross-section in the sine-wave direction then provides the one-dimensional spectral measurement $U(p, 0)$. Figure 4 shows an example of the computed signal modulation. Note that a peak-detection algorithm was used to identify the periodic spectral components and their amplitude. For the demonstrations in this paper care was taken when choosing the signal frequencies. An integer number of periods for each frequency component were included in the image region ( 2000 x 2000 pixels). 


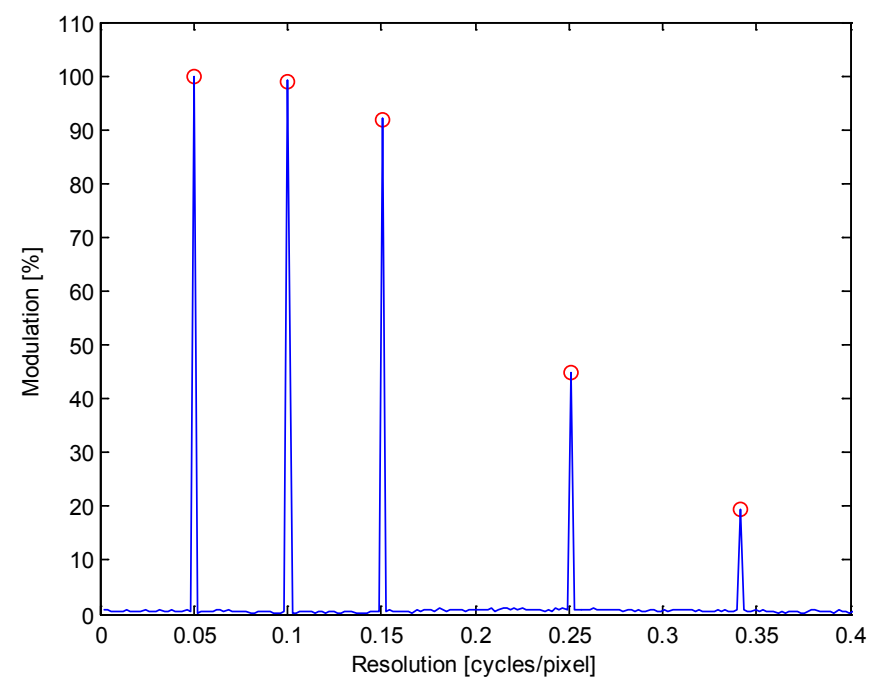

Figure 4: Example of the estimated modulation from the stacked sine-wave method and DFT analysis, for a five-frequency signal.

\section{GRID MULTI-BURST METHOD}

\subsection{Test Chart}

The classic IEC 61146-1 multi-burst chart was updated by reorganizing the frequencies into a $3 \times 3$ grid and adding OECF patches and alignment markers to enable the automatic detection and analysis. The OECF patches around midtone grey have $3 \%$ increments provide the sine-wave peak to peak reference amplitude for the normalization of the extracted sine-wave amplitudes. This normalization step is a key apart of the analysis of sine-wave based resolution targets. This chart is termed 'grid multi-burst' in this paper.

The purpose of simulating the image-capture of this chart is to provide a measurement reference for the stacked sinewave analysis method. A software chart generator was designed to produce the synthetic charts used in this study, an example of which is shown in Figure 5. For this test target, the spatial frequencies increase at the rate of the $\sqrt{2}$, which yields a 16x range in frequencies across the $3 \times 3$ grid. The sine-wave amplitudes were $3 \%$, and $6 \%$ of the maximum signal. The mean signal of the central texture region is $18 \%$.

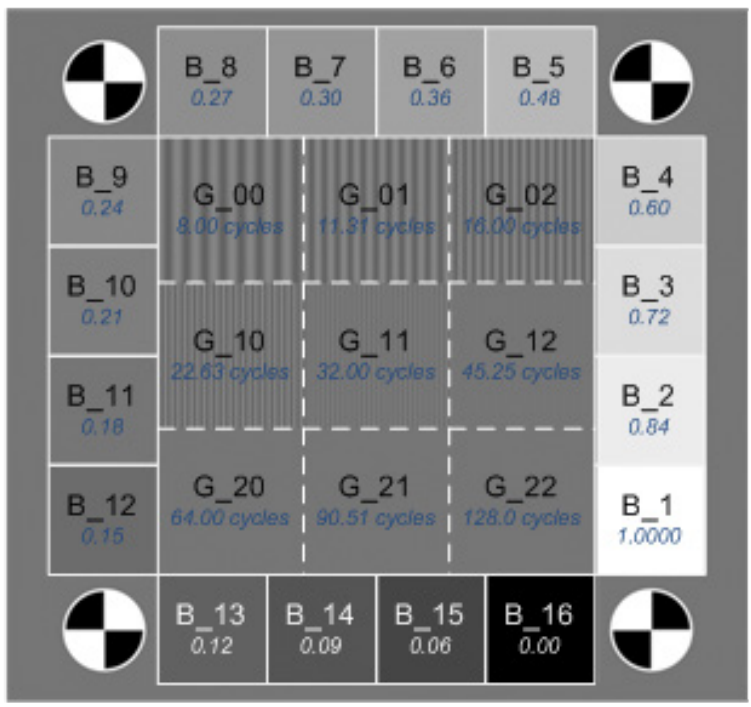

Figure 5: Multi-Burst inspired 3x3 grid of sine-waves with OECF and alignment mark features 


\subsection{Analysis Method}

Via the automatic finding of the checker alignment markers the location of the OECF patches, $3 \times 3$ grid cells and chart rotation is determined. After the sRGB linearization step, the OECF patch means are generated with the values near midtone used to determine the reference amplitude for the final normalization step. For each cell in the grid a sine-wave plane

$$
f(x, y)=a+b \sin (2 \pi(c x+d))
$$

is fitted to the 2-D data for that cell. The expected frequency values are used to seed the optimization algorithm minimization. An estimate of the original sine-wave (exposure) is used to normalize the fitted sine-wave amplitudes using the OECF. Note that the 3\% patch spacing matches both the 3\% and 6\% sine-wave amplitudes simulated.

\section{EMBEDDED SIGNAL CHART DESIGN}

As discussed above, the motivation for combining, or embedding, periodic signals with natural or computed image texture was the evaluation of systems with adaptive image processing. For the test signals to be valid indicators of underlying signal modulation, we concluded that a simple pixel-by-pixel addition of low-contrast periodic signals with the texture array was called for. We embedded both types of periodic signals; stacked and grid multi-burst, with two types of texture (natural and computed). A region of natural grass texture embedded with a multi-burst region is shown in the left panel of Figure 6. The corresponding test chart for a stacked sine-wave and computed dead-leaves texture region are shown in the right of Figure 6. The one-dimensional sine-wave signals were computed as functions of the x-, or row-direction.
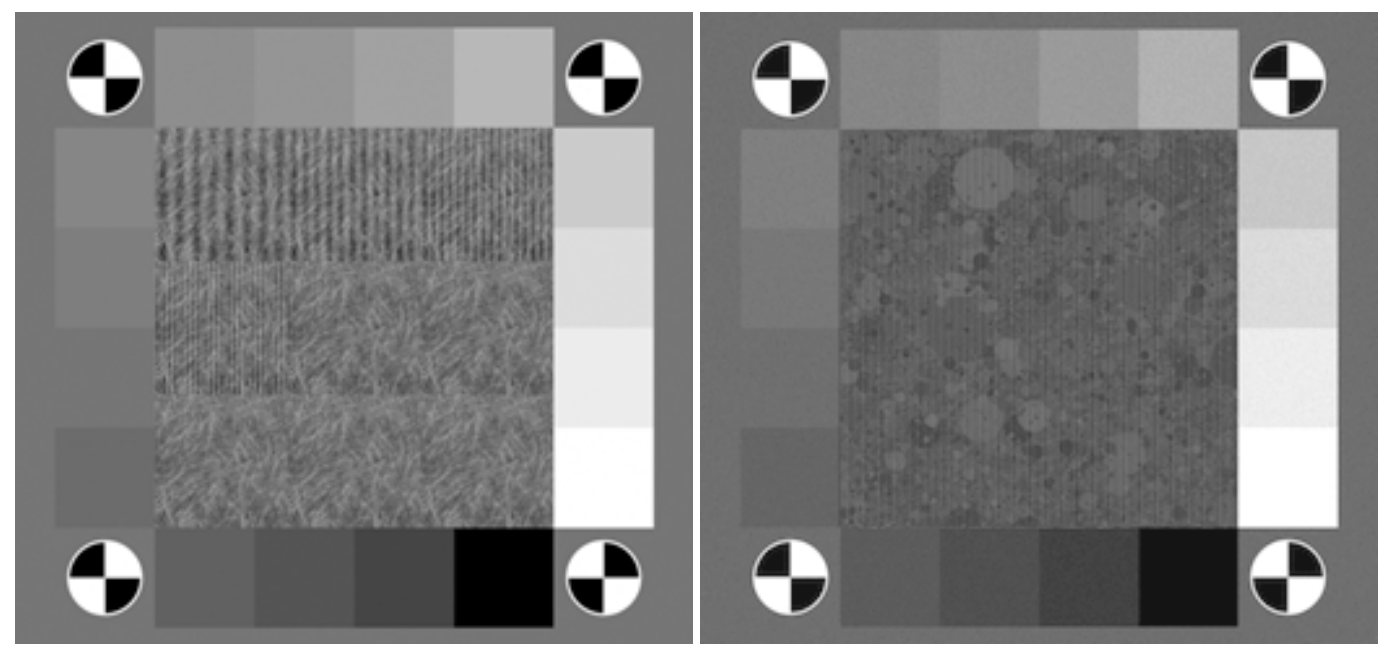

Figure 6: A grid multi-burst sine-wave chart with high-pass filtered version of the grass texture overlaid (left). A sixfrequency stacked sine-wave chart combined with deal-leaves target (right).

\section{CAMERA SIMULATION WITH ADDITIVE NOISE MODEL}

In order to test the two embedded sine-wave methods with various image noise levels, a simple camera image simulation was employed. The additive noise model is based on converting the signal to the equivalent electrons for a given ISO setting and adding the photon shot noise and readout noise contributions. The Poisson distribution was used for the photon shot noise. Photon shot noise has the characteristic that the noise deviation follows the square root of the signal in terms of electrons. For an APS-C sensor with $3.9 \mu \mathrm{m}$ pixel the maximum digital output codes at ISO100 represents a signal in the range of 30,000 to 40,000 electrons. The maximum signal that a digital output value represents is a function of ISO speed, e.g. ISO 25,600 has a maximum signal in electrons of $\sim 128 \mathrm{e}$. Table 1 shows the range of ISO values used, and the corresponding rms noise levels. 
The simulation assumptions were

- Maximum signal of 32,768e @ ISO100. Maximum signal in electrons is proportional to 1 over the ISO speed, for example if the ISO speed doubles the maximum signal in electrons is reduced by a factor of 2 .

- A 2.0e rms noise representing the readout noise of the image sensor. This does not vary with ISO Speed.

- 14-bit ADC (Standard for the latest generation of D-SLR cameras)

Table 1 illustrates the ISO speed to $18 \%$ noise relationship.

\begin{tabular}{|c|c|c|c|c|c|c|}
\hline \multirow{2}{*}{ ISO Speed } & \multirow{2}{*}{$\begin{array}{c}\text { Maximum } \\
\text { Signal [e] }\end{array}$} & \multirow{2}{*}{$\begin{array}{c}\text { Readout } \\
\text { Noise [e] }\end{array}$} & $\begin{array}{c}18 \% \text { Signal } \\
{[\mathrm{e}]}\end{array}$ & \multicolumn{3}{|c|}{ Noise @ 18\% } \\
\cline { 5 - 7 } & & & {$[\mathrm{e}]$} & {$[\%]$} & [codes] \\
\hline 100 & 32,768 & 2.0 & 5989 & 76.8 & 0.23 & 38.4 \\
\hline 400 & 8,192 & 2.0 & 1474 & 38.4 & 0.47 & 76.9 \\
\hline 1,600 & 2,048 & 2.0 & 368 & 19.3 & 0.94 & 154.4 \\
\hline 6,400 & 512 & 2.0 & 92 & 9.8 & 1.92 & 313.8 \\
\hline 25,600 & 128 & 2.0 & 23 & 5.2 & 4.06 & 665.6 \\
\hline
\end{tabular}

Table 1: ISO Speed versus 18\% RMS Noise relationship. The maximum signal in electrons is equivalent to maximum digital code value e.g. 255 for 8 bit or 16353 for 14-bit in the output image data

\subsection{Simulation flow}

An overview of the simulation flow used is provided in Figure 7. The synthetic charts were generated at 4 times the output resolution. Either a Gaussian blur filter (Figure 8 left) or a Butterworth Low pass filter (Fig 8 right) was applied prior to down sampling to provide a low-pass filter characteristic to image and avoid aliasing. The Gaussian blur low pass filtering step is a simple approximation for the blur induced by the optical low pass filter common in D-SLR cameras. An important point is that low pass optical blur creates a decrease in contrast (sine amplitude) as the spatial frequencies increases. Thus the input to the noise reduction is not constant contrast versus spatial frequency. To generate images with a wide region of constant contrast the Butterworth filter was used. After down sampling the above additive noise is added.

The image data input to the noise reduction filters as 14-bit linear RGB data. The Siemens Star and sine-wave fitting analysis is run on the 14-bit linear RGB data output from the noise reduction filters. After the noise filtering is completed the image is converted to 8-bit sRGB for visual inspection and comparison.

\section{MEASUREMENT STABILITY VERSUS NOISE \& TEXTURE}

The embedded sine-wave methods were evaluated for stability with respect to increased noise levels and effect of the addition of texture. Figure 9 and Figure 10 show the performance of the simulated images of stacked sine-wave and grid multi-burst sine-wave grid methods and the low contrast Siemens Star up to ISO 25.600 equivalent levels of noise. Both the stacked and grid multi-burst approaches are robust versus noise due to the fit working over the whole of the region or a $1 / 3 \times 1 / 3$ sub-region. As the fitting for the Siemens Star is performed over a smaller number of pixels, the circumference of the circle for the given spatial frequency, it is more sensitive to noise especially at the higher frequencies where number of samples is the least. 


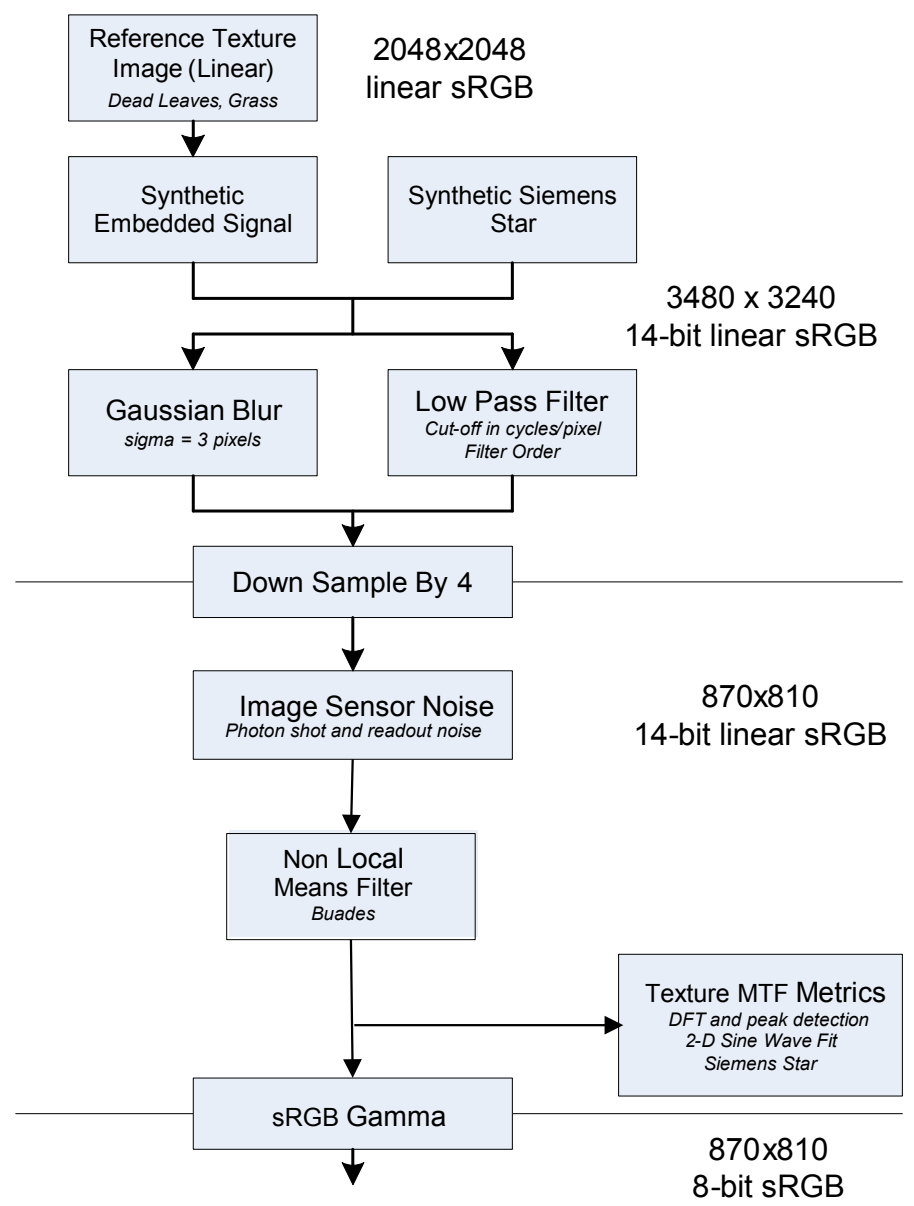

Figure 7: Outline of the simulation and image processing used to test the embedded sine-wave methods
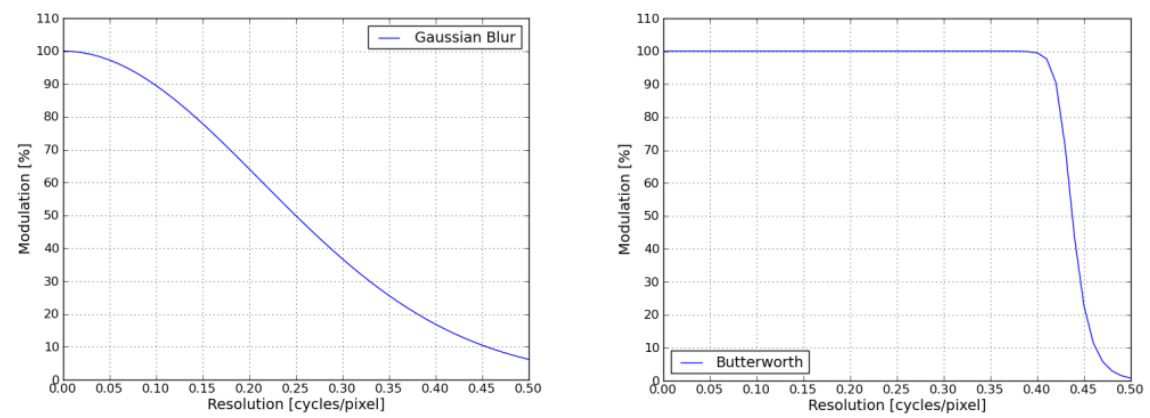

Figure 8: Gaussian blur response after down scale (left) Butterworth low-pass filter response. Cut-off frequency $=0.43$ cycles/pixel, filter order $=32$ (right) 

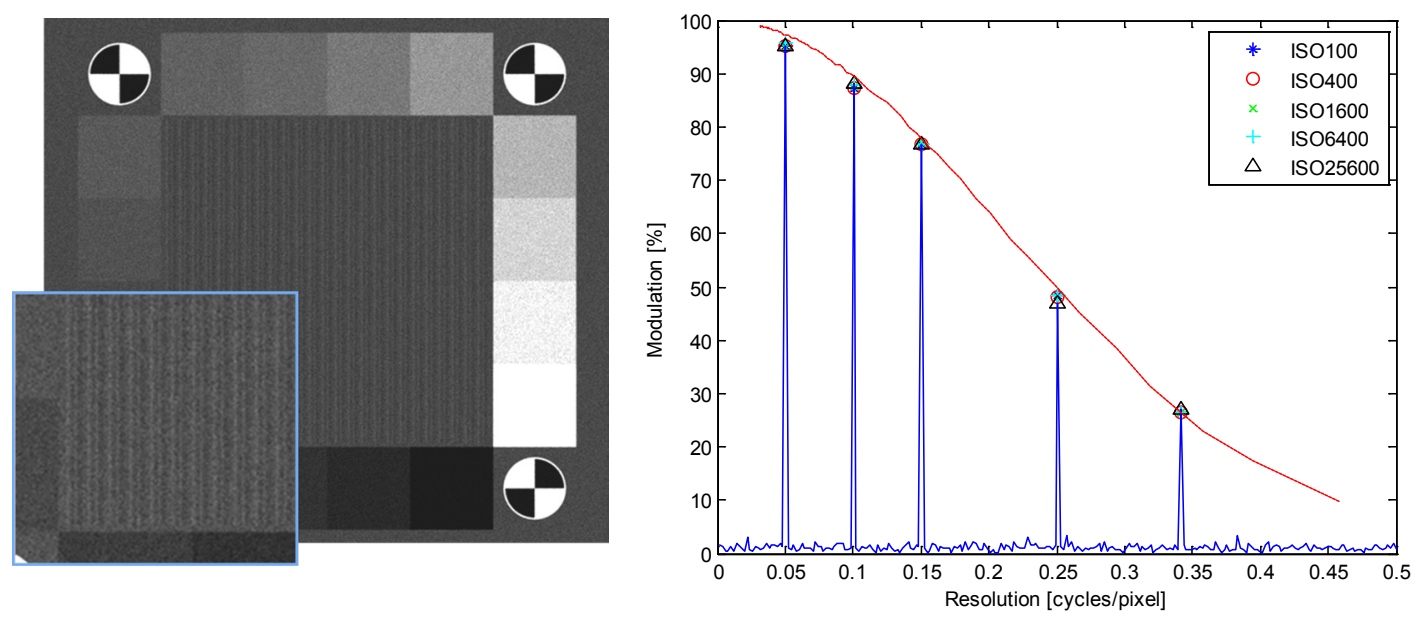

Figure 9: Simulated capture of stacked sine-wave target without texture, for ISO 25600, (left), and measured signal modulation for several ISO settings.
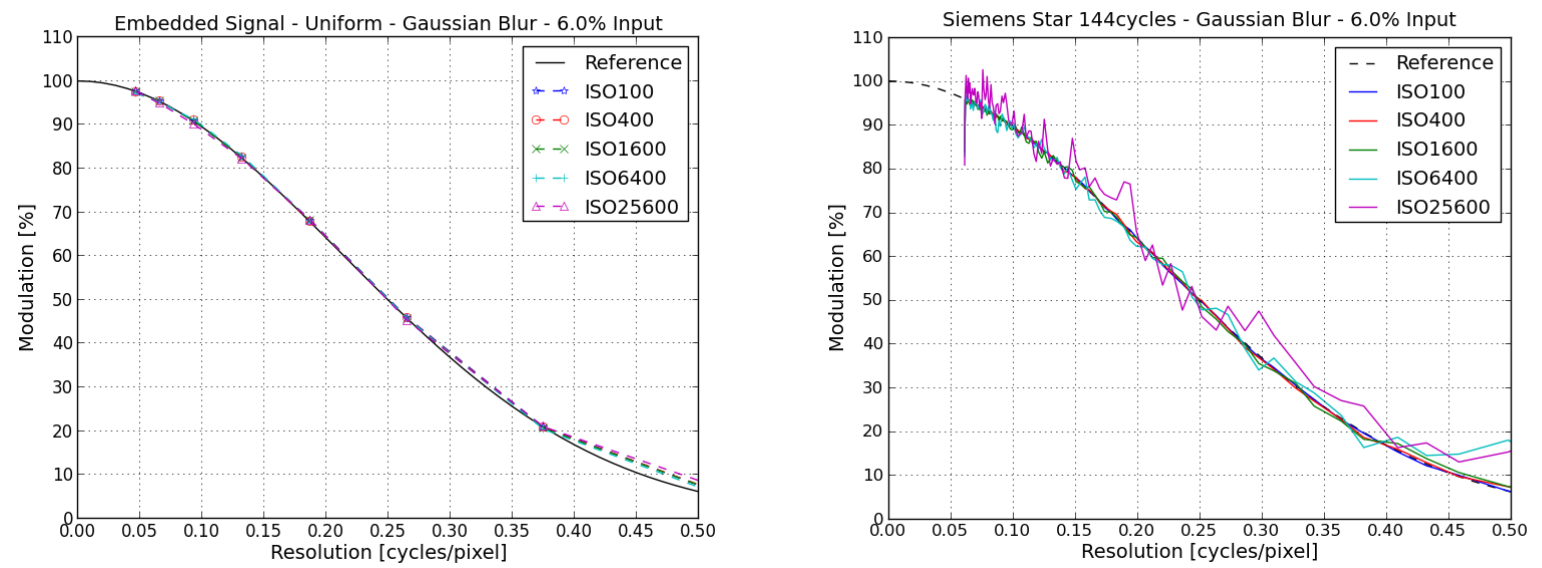

Figure 10: Measured signal modulation of grid multi-burst sine-wave target without texture, (left), and low contrast Siemens Star (right), both with $6 \%$ amplitude sine-wave for several ISO settings.

The signal modulation was also estimated for texture images with embedded signals. The components were successfully measured in both grass and deal-leaves texture fields. Figure 11 shows two representative results for relatively highcontrast signals, $6 \%$ and $4.5 \%$, respectively. On the left, the grid multi-burst sine-wave results are shown for the grass pattern and various simulated noise levels. Also shown, on the right, are results from the stacked sine-waves embedded in the dead-leaves texture pattern. Comparing Figs. 9 and 11 (right), we observe the increased low-frequency spectrum of the dead-leaves pattern. However, for these conditions, the dead-leaves spectra do not interfere with the measurement of the periodic spectral components. 

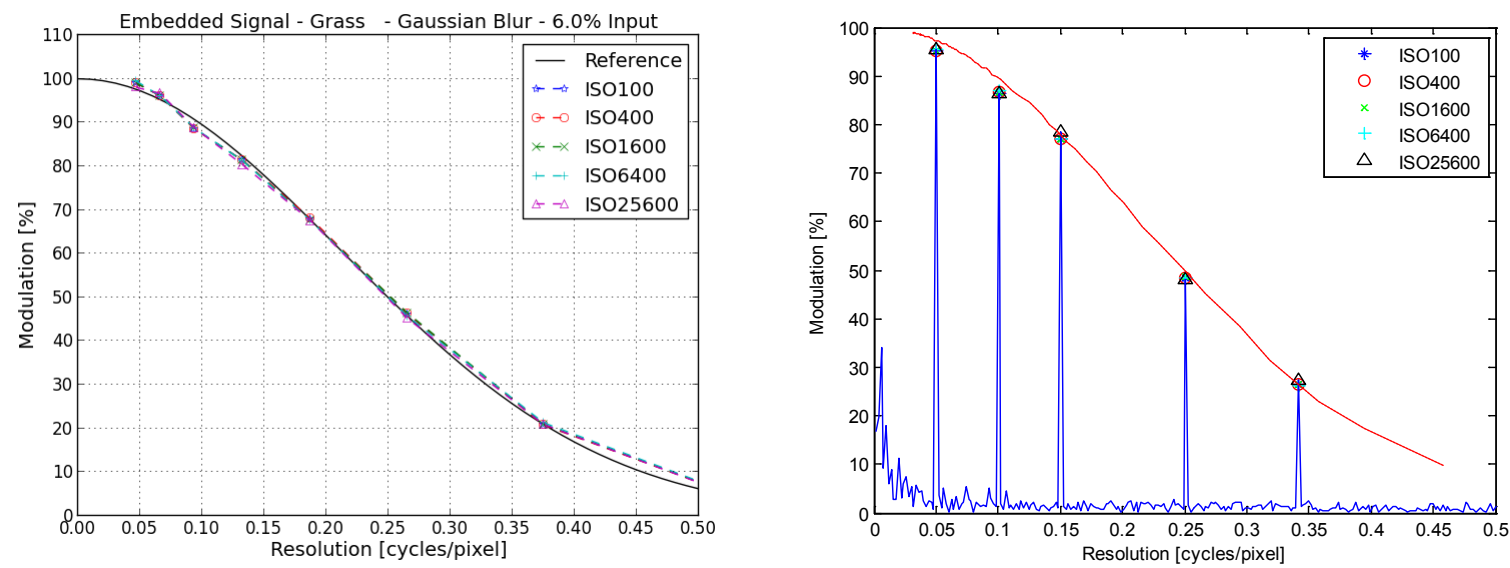

Figure 11: Measurement results for the grid multi-burst sine-wave chart with natural (grass) image content and stacked sinewave embedded signal with five components the dead-leaves texture (right).

\section{APPLICATION TO NON-LOCAL MEANS FILTERING}

The NL means filtering methods search for the pattern around the current pixel (the similarity window e.g. $5 \times 5$ or $7 \times 7$ ) within a wider search window (e.g. 11.x11, 21x21,49x49). Locations with high similarity are given higher weighting in noise averaging. The similarity search is how NL means filtering 'adapts' to the structure of image content. The MATLAB code published by Buades [5] was used for the NL means filtering. The search window was $21 \times 21$, the similarity window $7 \times 7$. The filter strength was set to the noise sigma for the $18 \%$ grey level for that ISO setting (Table 1).

Figure 12 shows the noise-power spectrum following NL filtering on a grass texture alone and a range of ISO (noise) levels. The most significant change in the power spectrum is above 0.025 cycles/pixel. This is consistent with size of the search window used. The low frequencies are left untouched. Thus frequencies $>0.025$ cycles/pixel seems to be relevant range for texture preservation assessment. When comparing the embedded stacked sine-wave and $3 \times 3$ sine-wave approaches, the stacked method yields the closest visual match to original texture after NL filtering (Figure 13)

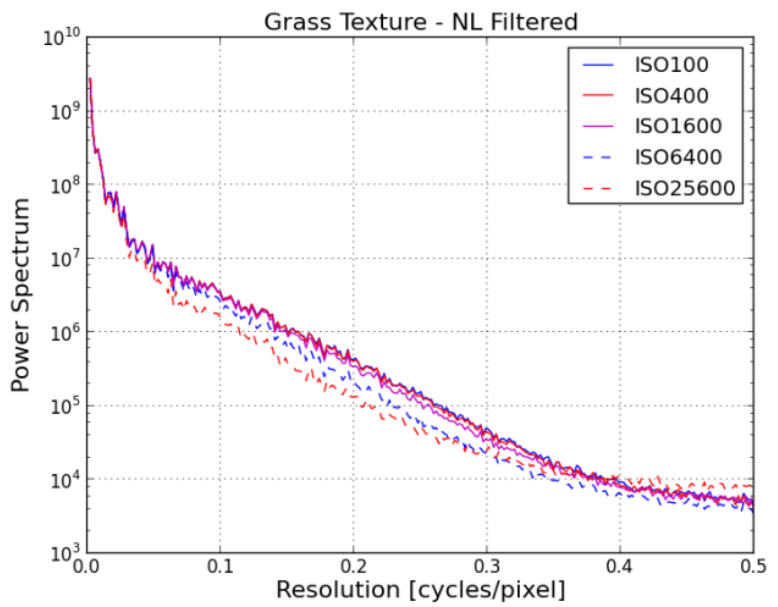

Figure 12: Power Spectra for NL Filtered Grass texture 

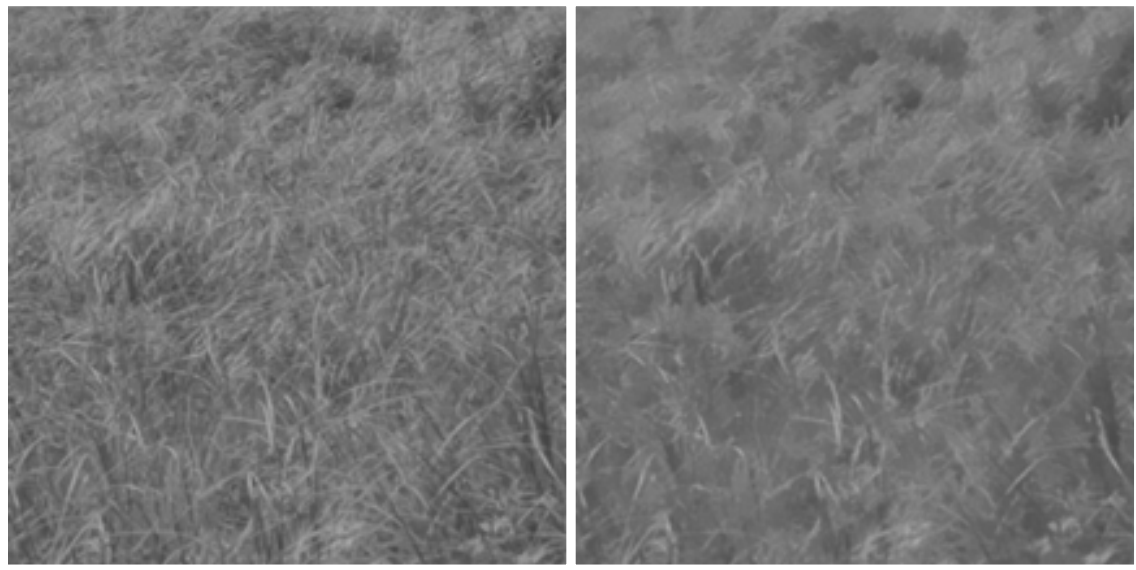

Figure 13: Grass Texture, ISO 400, filter strength $=76.8$ (left). Grass Texture, ISO 25,600, filter strength $=665.6$ (right)
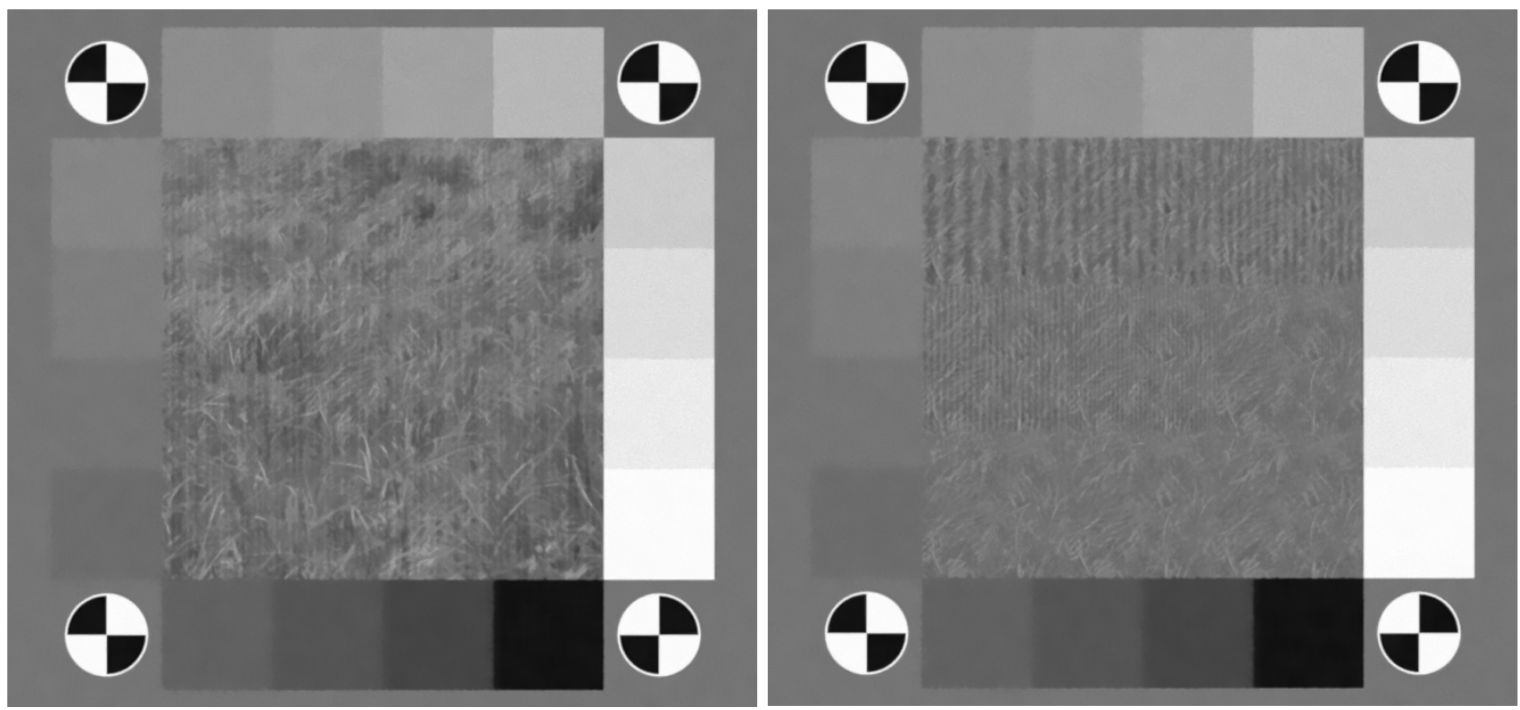

Figure 14: Embedded stacked sine-waves, grass texture, ISO 25,600, filter strength $=665.6$ (left). Embedded grid multiburst signal, grass texture ISO 25,600, filter strength $=665.6$ (right)

\subsection{Low Contrast Siemens Star NL filter Results}

To investigate the sensitivity to different contrast levels, synthetic low contrast Siemens Stars with sine-wave amplitudes of $3 \%, 6 \%$ and $9 \%$ were generated. The mean level of the star was $18 \%$. To avoid image clipping in the analysis sRGB linearization was used and the results normalized using the code range between the darkest and brightest OECF patches. Figure 15 shows the Siemens Star response to the constant contrast of Butterworth pre-filtered images with the application of the different NL means filter strengths in Table 1. The counter-intuitive result is that NL means filtering is good at preserving the sine-waves up to near the half-sampling (nyquist) frequencies, provided the contrast is larger than the filtering strength. It is only for ISO 6400 and beyond that attenuation is observed in the Siemens Star measurements. 

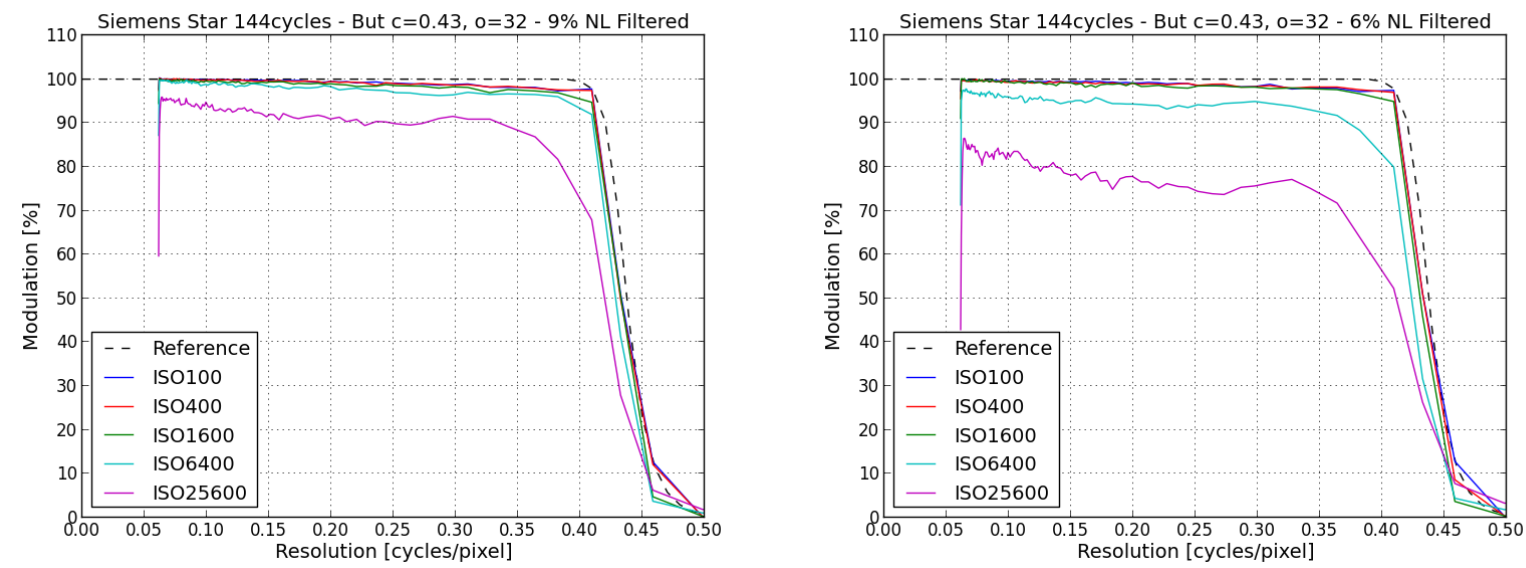

Figure 15: Siemens Start: 144 cycles 9\% amplitude (left) 6\% amplitude (right), Butterworth input image response

The Gaussian Blur pre-filtering step used in our simulation is better aligned with the low pass nature of camera optics. This is outlined in Fig. 16, where the lens MTF describes the optical blur. This is generally a decreasing function of spatial frequency. Therefore the input signal contrast level (sine-wave amplitude) for the noise-reduction operation decreases as the sine-wave feature frequency increases. Note that the modulation measurements shown in the right of Fig. 16 are for the Siemens Star pattern alone. No image texture is present.
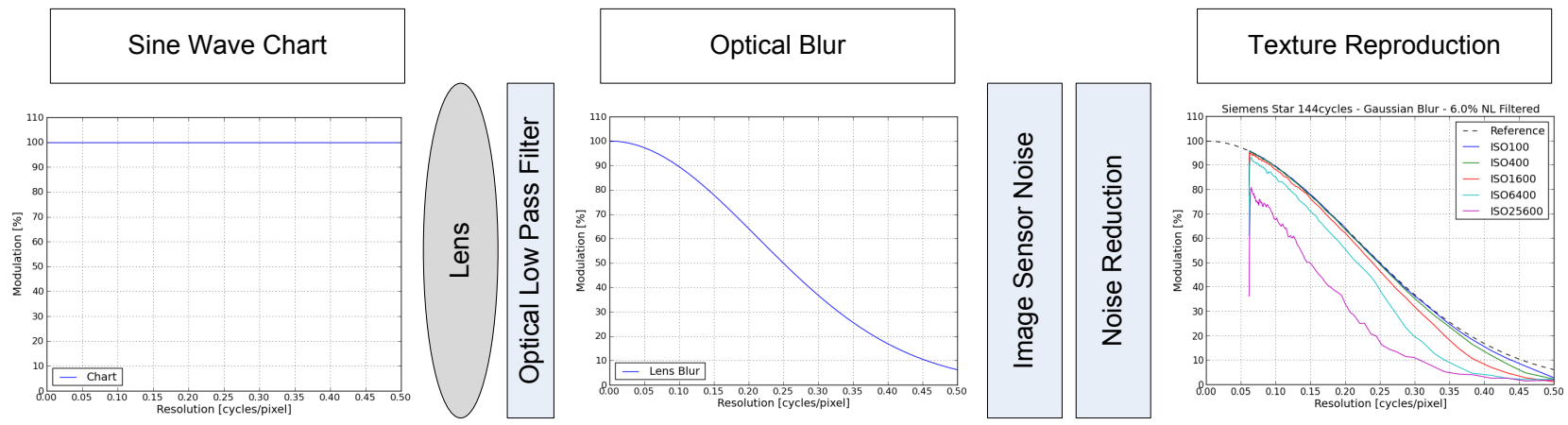

Figure 16: Influence of optical blur on the contrast versus spatial frequency characteristic at the input to the noise-reduction image processing operation

Figures 17 and 18 show the response to Gaussian blur pre-filtered images after the application of the different NL means filter strengths. As the sine-wave amplitude decreases from $9 \%$ to $3 \%$ the effect of NL means filtering becomes stronger. At the low ISO settings, the filter strength is small. For the low ISO settings only the high frequencies are attenuated as this is point where the contrast levels are the lowest. As the ISO setting increases the filter strength increases, the contrast levels over which the NL filter attenuates widens. NL means filters are primarily contrast driven.

Many of the 144 cycle Siemens Star measurements for the strong NL filtered levels do not attain $100 \%$ modulation at low frequencies. However the lower frequency range of a 72 cycle Siemens Stars reveals the rapid increase in the modulation at low frequencies which the 144cycles star fails to capture. A Siemens Star with $6 \%$ sine-wave amplitudes offers better sensitivity to texture loss than a $9 \%$ Star but avoids the sensitivity to noise of a $3 \%$ Star. 

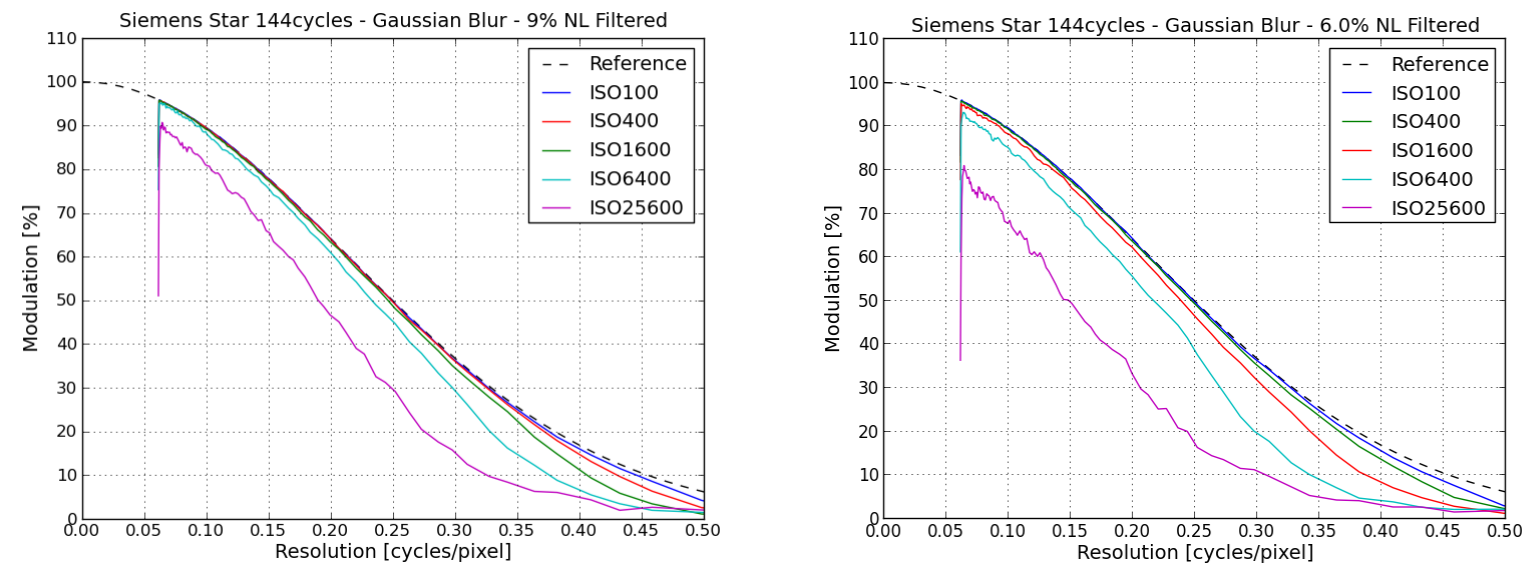

Figure 17: Siemens Star, 144 cycles, 9\% amplitude (left) 6\% (right), Gaussian Blur input image response and NL filtering
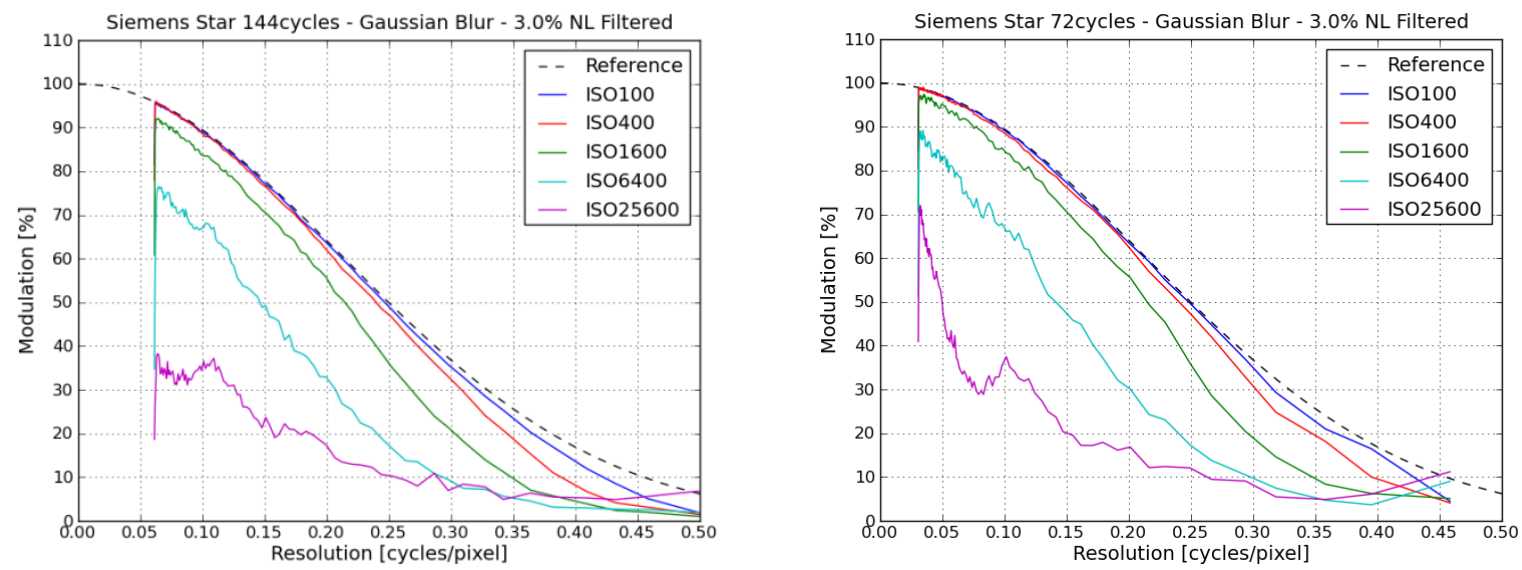

Figure 18: Siemens Star, 144 cycles (left) 72 cycles (right), 3\% amplitude, Gaussian Blur input image response.

\subsection{Sine-Wave Methods without Texture}

To provide a baseline for the evaluation of the proposed methods, NL means filtering was applied to simulated images of the grid multi-burst and stacked sine-wave charts without the addition of texture. Noise levels up to ISO 25,600 equivalent with the matching NL filter strength (Table 1) were simulated. The results are similar except at lower spatial frequencies the stacked approach has slightly lower response (Figure 19). A possible explanation is the lower sine-wave contrast of the stacked chart. 

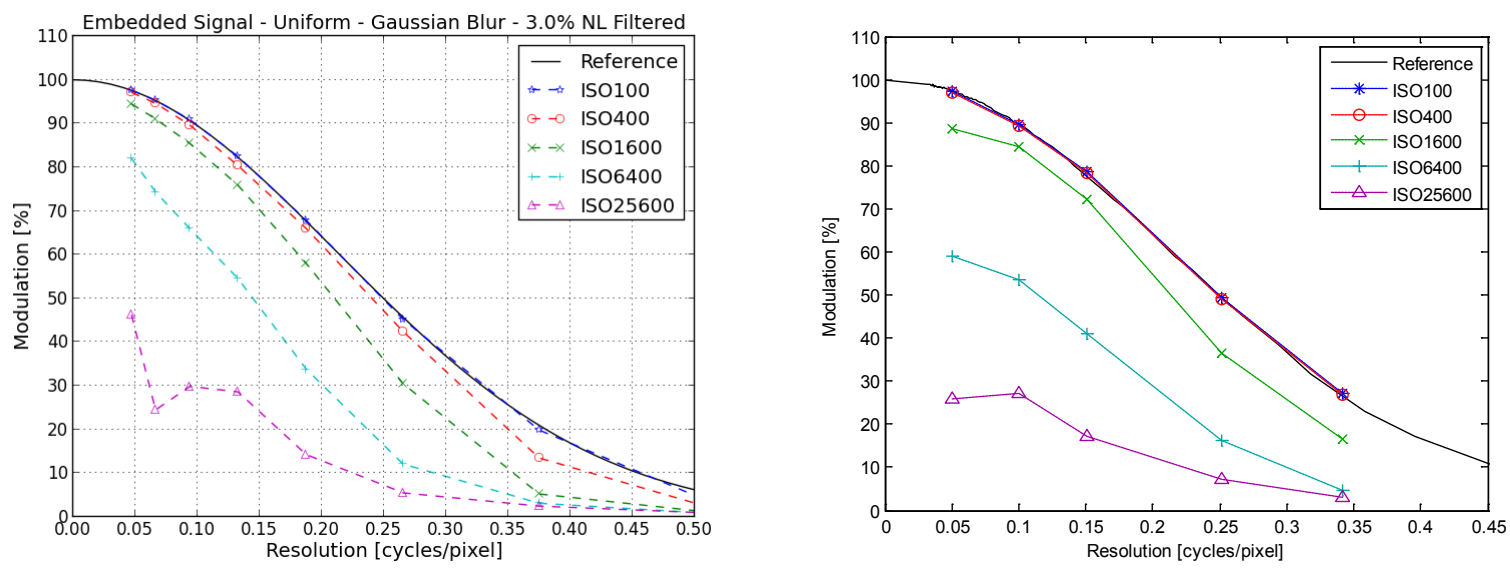

Figure 19: 3x3 grid sine-wave, 3\% amplitude (left), stacked sine-wave, 1.5\% amplitude (right), without texture, Gaussian blur input image response

\subsection{Embedded Signal Performance}

Figure 20 shows the effect of adding the texture to the simulated grid multi-burst and stacked sine-wave charts. The most noticeable delta is the raised response at lower spatial frequencies, suggesting the contrast level at these frequencies has been increased by the addition of the grass texture. The trends remain well matched for both the grid multi-burst and stacked sine-wave approaches. For the lowest two ISO settings the stacked sine-wave approach is a better match to the reference.
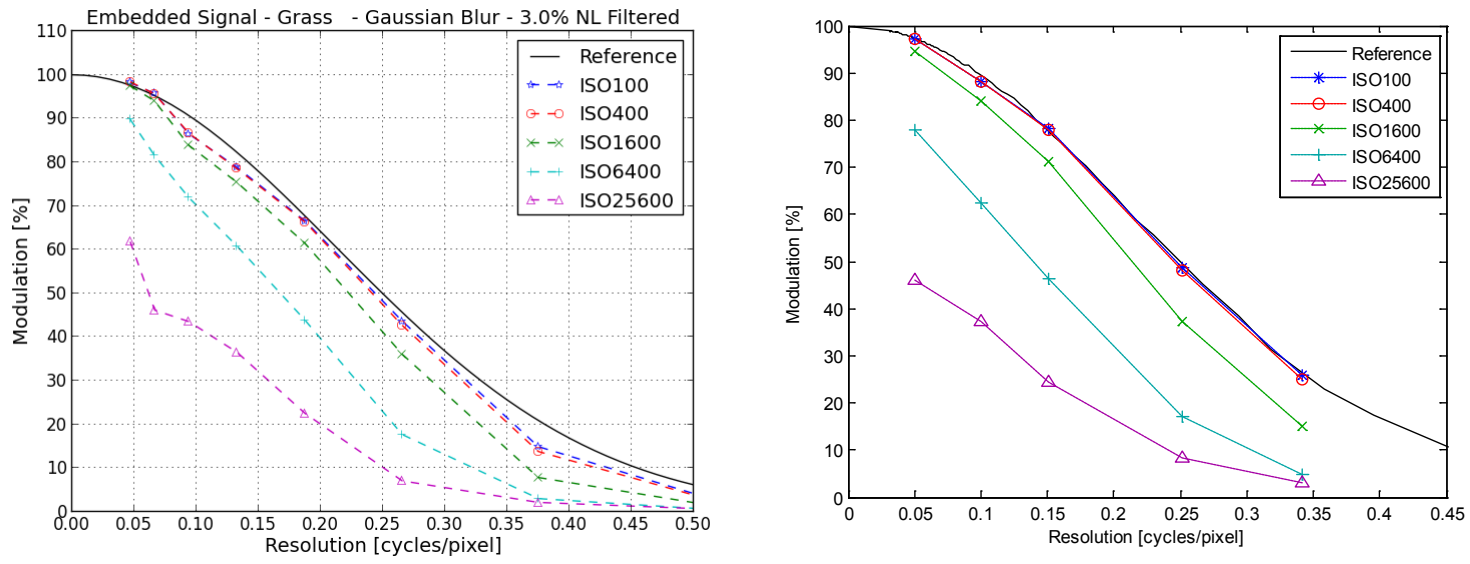

Figure 20: Results for the grid multi-burst method 3\% amplitude (left) stacked sine-wave method, 1.5\% amplitude (right) after non-local filtering, Gaussian blur input image response, embedded with grass pattern.

\section{SUMMARY}

We have reviewed the requirements for an improved texture measurement protocol, and described an alternative approach to texture reproduction analysis. This is based on embedding periodic test signals within texture regions. Two key requirements for texture reproduction metrics are: (1) the test target should have microstructure that is similar to natural textures, and (2) the analysis should be robust for high noise levels. A new method, based on the combining, or stacking of several sine-waves of different frequencies is described, as is the embedding in both natural and computed image texture. The stacked sine-waves were demonstrated and compared with another method for periodic signal embedding, the grid multi-burst. In addition these embedded-signal approaches were compared with the established Siemens Star method. For both embedded methods, the signals were chosen to be of low contrast so as not to influence the adaptive image processing parameters that would normally operate for natural scenes. 
For all three methods, synthetic test stimuli were generated using a Python-based camera simulation model. This model included a simple lens blur and ISO dependent noise model. Both embedded methods yielded stable texture-reproduction measures for a wide range of simulated exposure/noise levels. It appears, however, that the stacked method can be successfully used with a lower contrast than the grid method, closer to that of visual detection in a texture field. When this is the case, the appearance of corresponding texture regions with and without embedded signals is visually very similar. This is consistent with the above objectives for this work.

A non-local means filtering algorithm was used as the example of an adaptive noise-reduction algorithm for testing the embedded techniques. This algorithm has the important attribute of being driven by the local image contrast level, and is capable of preserving the texture of sine-waves if the contrast is higher than the filter strength. The stacked and $3 \times 3$ grid sine-wave approaches are shown to be robust to noise, and able to extract the correct reference (low-noise case) MTF profile for embedded texture. The blur generated by the application of NL means filtering to stacked sine-waves embedded in texture appeared very similar to processed regions of normal texture - a positive result. Corresponding regions for the $3 \times 3$ multi-burst appeared different, and therefore this method may be provide a less-effective texture measurement for camera paths that include similar filtering operations.

\section{REFERENCES}

[1] Cao, F., Guichard, F., and Hornung, H., "Measuring texture sharpness of a digital camera," Proc. SPIE 7250, $72500 \mathrm{H}(2009)$

[2] Burns, P. D., and Williams, D. "Measurement of Texture Loss for JPEG 2000 Compression," Proc. SPIE 8293, $82930 \mathrm{C}(2012)$

[3] Loebich, C., Wüller, D., Klingen, B., Jäger. A., "Digital Camera Resolution Measurement Using Sinusoidal Siemens Stars," Proc. SPIE 6502, 6502N (2007).

[4] Buades, A., Coll, B., and Morel, J., "A non-local algorithm for image denoising," Computer Vision and Pattern Recognition, 2, 60-65 (2005)

[5] McElvain, J., Campbell, S. P., Miller, J., and, Jin, E. W., "Texture-based measurement of spatial frequency response using the dead leaves target: extensions, and application to real camera systems," Proc. SPIE 7573, 75370D (2010)

[6] Schmidt, R.O, "Multiple Emitter Location and Signal Parameter Estimation," IEEE Trans. Antennas Propagation, AP-34, 276-280 (1986) 